\title{
Preparo da semente de colubrina para execução do teste de tetrazólio
}

\section{Preparation of colubrine seed for tetrazolium test}

\author{
Pablo Henrique Fernandes Moraes ${ }^{1}$, Laura Veríssimo Cavalcante ${ }^{2}$, Asclépio Silva de Albuquerque , $^{3}$ \\ Amanda Maria Carlos Rodrigues ${ }^{4}$, João Luciano de Andrade Melo Junior ${ }^{5}$, Luan Danilo Ferreira de \\ Andrade Melo ${ }^{6}$
}

${ }^{1}$ Graduando em agronomia, Universidade Federal de Alagoas, Centro de Ciências Agrárias, BR-104, Rio Largo, Alagoas. Fone: (82)996946227. E-mail: fernandesph2@gmail.com; ${ }^{2}$ Graduanda em agronomia, Universidade Federal de Alagoas, Centro de Ciências Agrárias. E-mail: lau_verissimo1@hotmail.com; ${ }^{3}$ Graduando em agronomia, Universidade Federal de Alagoas, Centro de Ciências Agrárias, E-mail: asaw777@ gmail.com; ${ }^{4}$ Aluna de graduação, Universidade Federal de Alagoas, Centro de Ciências Agrárias. E-mail: amandamaria.c.r@ gmail.com; ${ }^{5}$ Engenheiro Agrônomo, Doutor, Universidade Federal de Alagoas, Centro de Ciências Agrárias. E-mail: luciiano.andrade@ yahoo.com.br; ${ }^{6}$ Engenheiro Agrônomo, Doutor, Universidade Federal de Alagoas, Centro de Ciências Agrárias. E-mail: luan.danilo@yahoo.com.br.

\begin{tabular}{l} 
A R T I G O \\
\hline Recebido: 06/11/2019 \\
Aprovado: 27/11/2019 \\
\hline Palavras-chave: \\
Caatinga \\
Colubrina glandulosa \\
Períodos de coloração \\
Viabilidade de sementes
\end{tabular}

Key words:

Caatinga

Colubrina glandulosa

Staining periods

Viable seeds

\begin{abstract}
R E S U M O
Colubrina glandulosa Perkins, conhecida popularmente como sobrasil, é uma espécie de importância ecológica e socioeconômica, porém ameaçada de extinção. Suas sementes apresentam germinação baixa, lenta e desuniforme. Sabendo que o teste de tetrazólio tem se destacado por sua eficácia e rapidez quando se deseja respostas rápidas sobre a qualidade das sementes, este estudo objetivou estabelecer a melhor concentração da solução de tetrazólio e o período de coloração para avaliar a viabilidade das sementes de Colubrina glandulosa. As sementes foram pré-umedecidas em papel toalha e colocadas em uma câmara de germinação a $30{ }^{\circ} \mathrm{C}$ por cinco horas. Posteriormente, a porção do cotilédone contendo o embrião foi imersa em solução de 2,3,5-trifenil cloreto de tetrazólio em quatro concentrações de 0,$075 ; 0,1 ; 0,5$ e $1,0 \%$ por três períodos de coloração $\left(2,4\right.$ e 6 horas) a $30{ }^{\circ} \mathrm{C}$ no escuro. $\mathrm{O}$ delineamento experimental utilizado foi o inteiramente casualizado, em esquema fatorial 4 x $3+1$ (4 concentrações de solução de tetrazólio x 3 períodos de coloração +1 controle - teste de germinação). O teste de tetrazólio foi eficiente para estimar a viabilidade de sementes de $C$. glandulosa, e a concentração de $0,075 \%$ por quatro horas a $30^{\circ} \mathrm{C}$ foi a melhor condição.
\end{abstract}

A B S T R A C T
The Caatinga ecoregion has a great diversity of species with exploitation potential. Among
them, colubrina (Colubrina glandulosa Perkins) stands out for its importance in logging and
forestry activities. Its seeds have low, slow and uneven germination. Knowing that the use of
the tetrazolium test to estimate viability becomes essential when rapid response on seed quality
is desired, this study aimed to establish the best tetrazolium solution concentration and staining
period to evaluate the viability of Colubrina glandulosa seeds. Initially, the seeds were
previously moistened between sheets of paper for five hours at $30^{\circ} \mathrm{C}$. Subsequently, the portion
of the cotyledon containing the embryo was immersed in four tetrazolium solution
concentrations $(0.075,0.1,0.5$, and $1.0 \%)$ and three staining periods $(2,4$, and 6 hours) in the
dark. under the temperature of $30{ }^{\circ} \mathrm{C}$. The percentage of viable seeds was compared with the
results obtained in the germination test performed on the paper substrate at $30{ }^{\circ} \mathrm{C}$ in four
replications of 25 seeds. The tetrazolium test was efficient to estimate the viability of $C$.
glandulosa seeds, and the concentration of $0.075 \%$ for four hours at $30{ }^{\circ} \mathrm{C}$ was the best
condition.

geográfica, encontrada em diversos biomas do país (BRANCALION et al., 2011).

O método de propagação de $C$. glandulosa é por reprodução sexuada, sendo indispensável a avaliação da qualidade fisiológica das sementes. Sementes desta espécie são consideradas de comportamento ortodoxo, com poder germinativo alto quando a sua dormência é superada (CARVALHO, 2005).

\section{Revista Verde}

ISSN 1981-8203

Pombal, Paraíba, Brasil v. 14, n.5, Edição Especial, p.625-628, 2019 doi: 10.18378/rvads.v14i5.7599 
O teste de germinação é a forma mais frequente para obtenção de resultados quanto à qualidade fisiológica das sementes, conduzido sob condições controladas de umidade, temperatura e fotoperíodo. Por outro lado, muitas vezes não reflete o comportamento das espécies no campo, além do que, demanda um período de tempo relativamente maior para a obtenção dos resultados (NOGUEIRA et al., 2014).

O teste de tetrazólio tem se destacado por sua eficácia e rapidez. Trata-se de um teste bioquímico, baseado na atividade das enzimas desidrogenases envolvidas no processo de respiração. Pela hidrogenação do 2-3-5-trifenil cloreto de tetrazólio, é produzida nas células vivas uma substância vermelha, o trifenil formazan. Com a reação, é possível diferenciar as partes vivas, coloridas de vermelho, daquelas mortas, que possuem a cor branca-leitosa (SOUSA et al., 2017).

Os parâmetros adotados para a sua aplicação prática, como concentração da solução do sal de tetrazólio e o tempo de exposição para a coloração, divergem quanto a espécie a ser testada, do método de preparo das sementes e da permeabilidade do tegumento (DANTAS et al., 2015).

A aplicação do teste de tetrazólio restringe-se, principalmente, às sementes de amplo uso agrícola como soja, milho, hortaliças e gramíneas forrageiras, porém, a crescente demanda por sementes de espécies florestais ressalta a necessidade em se desenvolver métodos para a avaliação da viabilidade dessas sementes (PINTO et al., 2008).

Nas Regras para Análise de Sementes (BRASIL, 2009) e nas Instruções para Análise de Sementes de Espécies Florestais (BRASIL, 2013) que, ainda, encontra-se em fase de atualização, há carência de informações específicas sobre a ecofisiologia da germinação de sementes dessa espécie, que ainda não tem os critérios estabelecidos para a padronização dos métodos de análise, tendo em vista a produção de mudas.

Com base no exposto, este trabalho teve como objetivo estabelecer a melhor concentração de solução de tetrazólio e período de coloração para avaliar a viabilidade de sementes de C. glandulosa.

\section{MATERIAL E MÉTODOS}

A pesquisa foi desenvolvida no Laboratório de Propagação de Plantas do Campus de Engenharias e de Ciências Agrárias, na cidade de Rio Largo, Alagoas, Brasil, com sementes de $C$. glandulosa coletadas de dez matrizes pertencentes a fragmentos de Caatinga, na cidade de Bom Conselho, PE (9॰ 10' 11 "S; 36 40' 47" W e 654 m de altitude) de outubro a dezembro de 2018.

Antes de realizar os experimentos, as sementes foram acondicionadas em frasco de vidro e armazenadas em câmara seca (16-18 ${ }^{\circ} \mathrm{C}$ e $43-45 \%$ UR do ambiente).

A determinação do teor inicial de umidade das sementes foi realizada pelo método de estufa a $105^{\circ} \mathrm{C} \pm 3{ }^{\circ} \mathrm{C}$ por 24 horas, utilizando duas repetições de um grama de sementes e os resultados expressos em porcentagem (base úmida) (BRASIL, 2009).

Para o teste de tetrazólio, as sementes de C. glandulosa foram pré-umedecidas em papel toalha e colocadas em uma câmara de germinação a $30^{\circ} \mathrm{C}$ por cinco horas. Em seguida, com bisturi, as sementes foram cortadas longitudinalmente e transversalmente, com a porção do cotilédone contendo o embrião, colocada em copos plásticos de $50 \mathrm{~mL}$ e imersa em uma solução de 2,3,5-trifenil cloreto de tetrazólio em quatro concentrações de 0,$075 ; 0,1 ; 0,5$ e $1,0 \%$ por três períodos de coloração (2, 4 e 6 horas) em uma câmara regulada a uma temperatura de $30{ }^{\circ} \mathrm{C}$ no escuro. Foram utilizadas quatro repetições de 25 sementes para cada combinação de concentração da solução de tetrazólio e período de coloração.

Após o período de coloração, a solução foi drenada, o material lavado em água corrente e os embriões foram extraídos da parte restante do cotilédone e mantidos em água em ambiente refrigerado até avaliação. Posteriormente, os embriões foram observados individualmente com microscópio estereoscópio e avaliados quanto à uniformidade, intensidade de cor, presença de áreas brancas leitosas, aparência dos tecidos e localização dessas colorações em relação às áreas essenciais do embrião (eixo hipocótilo-radicular e área vascular), sendo classificados em viáveis e inviáveis (AOSA, 2009).

Para comparar os resultados obtidos no teste de tetrazólio, o teste de germinação foi realizado em câmara de germinação do tipo B.O.D. regulada a temperatura de $30^{\circ} \mathrm{C}$ sob luz branca constante, com quatro repetições de 25 sementes. As sementes foram distribuídas sobre duas folhas de papel toalha umedecidas em água equivalente a 2,5 vezes a massa do substrato seco e colocadas dentro de caixas de plásticos transparentes para manter a umidade. $\mathrm{O}$ critério utilizado para a germinação das sementes foi o desenvolvimento de mudas normais, e a avaliação foi realizada no décimo nono dia após a semeadura (BRASIL, 2009).

O delineamento experimental utilizado foi o inteiramente casualizado, em esquema fatorial 4 × $3+1$ (4 concentrações de solução de tetrazólio x 3 períodos de coloração +1 controle teste de germinação). Sequencialmente, a análise de variância foi realizada pelo teste $\mathrm{F}$ com significância de $1 \%$ e $5 \%$ e as médias de sementes viáveis obtidas pelo teste de tetrazólio foram comparadas pelo teste de Tukey $(p \leq 0,05)$. A comparação entre as médias de sementes viáveis para cada uma das combinações no teste de tetrazólio com os resultados do teste de germinação (controle) foi realizada pelo teste de Dunnett $(\mathrm{p} \leq 0,05)$. As análises estatísticas foram realizadas no software ASSISTAT versão 7.6 beta (SILVA; AZEVEDO, 2002).

\section{RESULTADOS E DISCUSSÃO}

Os resultados para a porcentagem de sementes viáveis pelo teste de tetrazólio em diferentes concentrações e tempos de exposição e o teste de germinação (controle) para experimentos realizados na temperatura de $30{ }^{\circ} \mathrm{C}$ foram apresentados na Tabela 1. Houve um efeito significativo ( $\mathrm{p}$ $0,05)$ da interação entre a concentração da solução e o período de coloração para experimentos conduzidos a temperaturas de $30{ }^{\circ} \mathrm{C}$. Além disso, houve uma diferença significativa entre as estimativas de viabilidade das sementes obtidas pelo teste de tetrazólio e os resultados do teste de germinação, como pode ser visto pela significância do controle (porcentagem de germinação) versus contraste fatorial. 
Tabela 1. Porcentagem de sementes viáveis de $C$. glandulosa obtidas pelo teste de tetrazólio, em diferentes concentrações $(0,075$;

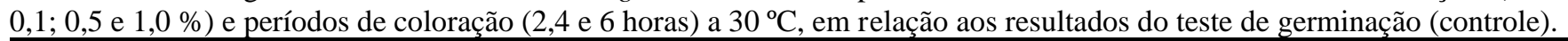

\begin{tabular}{|c|c|c|c|c|}
\hline \multirow{2}{*}{ Período de coloração (horas) } & \multicolumn{4}{|c|}{ Concentração de solução de tetrazólio (\%) } \\
\hline & 0,075 & 0,1 & 0,5 & 1,0 \\
\hline 2 & $56 \mathrm{bBy}$ & $87 \mathrm{aBy}$ & $33 \mathrm{cBy}$ & 32 cAy \\
\hline 4 & $98 \mathrm{aAz}$ & $99 \mathrm{aAz}$ & $67 \mathrm{bAy}$ & $31 \mathrm{cAy}$ \\
\hline 6 & $41 \mathrm{aCy}$ & $32 \mathrm{bCy}$ & $17 \mathrm{cCy}$ & $6 \mathrm{dBy}$ \\
\hline \multicolumn{5}{|c|}{ Germinação $=99 \mathrm{z}$} \\
\hline Valor de "F" para períodos (P) & & $1298,08 * *$ & & \\
\hline Valor de "F" para concentrações $(C)$ & & $778,80 * *$ & & \\
\hline Valor de "F" para interação (P x C) & & $86,65 * *$ & & \\
\hline Valor de " $F "$ para adicional vs fatorial & & $1090,12 * *$ & & \\
\hline Valor de "F" para tratamentos & & $532,14 * *$ & & \\
\hline $\mathrm{CV}(\%)$ & & 5,32 & & \\
\hline
\end{tabular}

Médias seguidas de mesma letra minúscula na linha (a, b, c, d) e maiúscula na coluna (A, B, C) não diferem a $5 \%$ de probabilidade pelo teste de Tukey. Médias seguidas de mesma letra $(z, y)$ entre a germinação (controle - teste de germinação) e a viabilidade obtida no teste de tetrazólio não diferem a $5 \%$ de probabilidade pelo teste de Dunnett.

Sob a temperatura de $30^{\circ} \mathrm{C}$, o período de quatro horas de coloração apresentou a maior estimativa de viabilidade, independentemente da concentração da solução de tetrazólio, exceto $1,0 \%$, cuja estimativa não diferiu do período de 2 horas, e valores mais altos para os períodos de coloração foram observados na concentração de $0,1 \%$ O período de quatro horas nas concentrações de $0,075 \%$ (98\% de sementes viáveis) e $0,1 \%$ (99\% de sementes viáveis) foi favorável para a avaliação das sementes, fornecendo estimativas semelhantes às dos resultados do teste de germinação (99\%). Quanto às demais combinações de períodos e concentrações, houve diferença entre estas e o controle, devido à coloração inadequada, que subestimou a viabilidade das sementes (Tabela 1).

Fogaça et al. (2006), Nogueira et al. (2014) e Sousa et al. (2017), testando diferentes concentrações da solução de tetrazólio em espécies florestais, observaram resultados que corroboraram com os obtidos no presente trabalho, onde a concentração de $0,075 \%$ apresentou eficiência na coloração das sementes. Segundo Silva et al. (2016), menores concentrações da solução reduziram os custos de implantação do teste, não afetando a sua eficácia quanto a identificação de injúrias.

Cunha e Gomes (2015) sugeriram como procedimento para condução do teste de tetrazólio de sementes de Erythrina velutina, a imersão por 3 horas na concentração de $0,075 \%$, porém não diferiu significativamente dos resultados alcançados no teste de germinação. Pinto et al. (2008) recomendaram a concentração de $0,075 \%$ em tempo menor de 90 minutos, quando submeteram sementes de Poecilanthe parviflora a $40^{\circ} \mathrm{C}$ no escuro.

Fogaça et al. (2011), estudando espécies florestais, constataram que a imersão por 4 horas na concentração de $0,1 \%$, proporcionou resultados satisfatórios quando da diferenciação de tecidos, e da qualidade fisiológica quando comparadas com o teste de germinação padrão.

Os padrões de coloração observados nos embriões variaram de rosa claro em sementes viáveis a branco leitosa em sementes mortas. Os embriões viáveis exibiram cores rosa claro ou vermelha ao longo de todo o comprimento, demonstrando que os tecidos estavam vivos e vigorosos. Por outro lado, embriões inviáveis, quando expostos à solução de tetrazólio, exibiam uma coloração vermelha intensa (deterioração do tecido) ou branco leitosa (tecido morto) em toda a sua extensão, ou áreas descoloridas (Figura 1).
Figura 1. Sementes viáveis de C. glandulosa: embrião com coloração rosa claro (A, B) e vermelha (C, D). Sementes inviáveis de C. glandulosa: embrião com áreas descoloridas (E), embrião com coloração vermelha intensa em toda a sua extensão $(\mathrm{F}, \mathrm{G})$ e branco leitosa $(\mathrm{H})$.
A

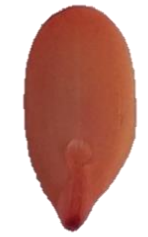

E

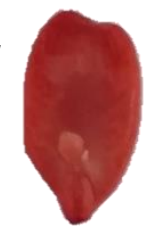

B
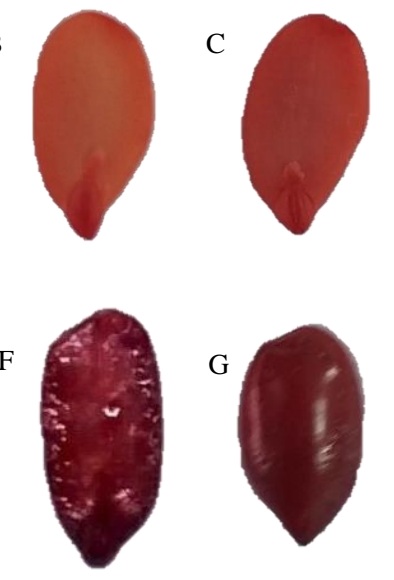

$\mathrm{D}$

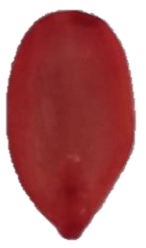

$\mathrm{H}$

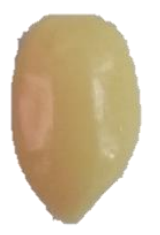

A escolha do método apropriado para o teste de tetrazólio deve basear-se na facilidade de identificação de tecidos viáveis e inviáveis e na capacidade de diferenciar o vigor das sementes. As diferenças de cores observadas nas sementes após a coloração em solução de tetrazólio são as principais características que devem ser consideradas na interpretação dos resultados dos testes (SANTOS; VIEIRA; PANOBIANCO, 2019).

A intensidade da cor das sementes no teste de tetrazólio varia entre as espécies. Nesta espécie, a cor que indica um tecido viável é vermelha ou rosa claro. Isso ocorre porque a terminologia usada para determinar as cores observadas nas sementes no teste de tetrazólio é geralmente estabelecida pelos autores. Portanto, elas podem variar entre os estudos.

$\mathrm{Na}$ avaliação do teste de tetrazólio de sementes de $C$. glandulosa à temperatura de $30{ }^{\circ} \mathrm{C}$, foram observadas variações na coloração das sementes de acordo com os tratamentos utilizados, e o período de coloração de 4 horas foi eficaz para avaliar a viabilidade das sementes desta espécie em concentrações de 0,075 e $0,1 \%$, pois proporcionaram uma coloração clara dos embriões, facilitando a análise e interpretação dos resultados (Figuras 1A, 1B, 1C e 1D). 
A exposição de embriões a uma concentração de solução de tetrazólio a 1,0\%, associado a um período de coloração de 6 horas, foi menos eficiente na classificação de sementes viáveis, por apresentar problemas para a coloração do embrião, dificultando a interpretação dos resultados (Figuras 1F, $1 \mathrm{G}$ e $1 \mathrm{H})$.

Também foi observado que em muitos embriões expostos nas concentrações de 0,5 e 1,0\%, uma intensa coloração vermelha se desenvolveu nas extremidades da plúmula ou no fundo do eixo hipocótilo-radícula (Figura 1F). Nesse caso, embriões que não desenvolveram coloração ideal podem ter sido considerados inviáveis quando a ausência de coloração poderia ter sido causada pela temperatura de incubação, combinada com diferentes concentrações e tempos.

Sementes de Leucaena leucocephala submetidas a concentração de $1,0 \%$ em diferentes períodos de tempo, apresentaram coloração excessiva, dificultando a interpretação do teste (COSTA; SANTOS, 2010).

\section{CONCLUSÕES}

A concentração de sal de tetrazólio a $0,075 \%$ por quatro horas a $30{ }^{\circ} \mathrm{C}$ é uma combinação eficaz para avaliar a viabilidade das sementes de $C$. glandulosa.

\section{AGRADECIMENTOS}

Ao Laboratório de Fitotecnia e ao Laboratório de Sementes Crioulas do Campus de Engenharias e Ciências Agrárias da UFAL.

\section{REFERÊNCIAS}

AOSA. Association of Official Seed Analysts. Seed vigor testing handbook. Ithaca, 2009. $341 \mathrm{p}$.

BRANCALION, P. H. S.; MONDO, V. H. V.; NOVEMBRE, A. D. L. C. Escarificação química para a superação da dormência de sementes de saguaraji-vermelho (Colubrina glandulosa Perk. - Rhamnaceae). Revista Árvore, v. 35, n. 1, p. 119-124, 2011. 10.1590/S0100-67622011000100014.

BRASIL. Ministério da Agricultura, Pecuária e Abastecimento. Instruções para análise de sementes de espécies florestais. Ministério da Agricultura, Pecuária e Abastecimento. Secretaria de Defesa Agropecuária/Coordenação Geral de Apoio Laboratorial. Brasília, DF: MAPA/SDA/CGAL, 2013. 97 p.

BRASIL. Ministério da Agricultura, Pecuária e Abastecimento. Regras para análise de sementes. Ministério da Agricultura, Pecuária e Abastecimento. Secretaria de Defesa Agropecuária. Brasília, DF: MAPA/ACS, 2009. 395 p.

CARVALHO, P. E. R. Sobrasil. Colombo: Embrapa Florestas, 2005. 10 p. (Embrapa Florestas. Circular Técnica, 106).

COSTA, C. J.; SANTOS, C. P. Teste de tetrazólio em sementes de leucena. Revista Brasileira de Sementes, v. 32, n. 2, p. 6672, 2010. 10.1590/S0101-31222010000200008.
CUNHA, M. C. L.; GOMES, I. H. R. A. Viabilidade de sementes de Erythrina velutina Willd pelo teste de tetrazólio. Pesquisas Agrárias e Ambientais. Nativa, v. 3, n. 3, p. 196-200, 2015.

DANTAS, B. F.; MATIAS, J. R.; RIBEIRO, R. C. Teste de tetrazólio para avaliar viabilidade e vigor de sementes de espécies florestais da Caatinga. Informativo Abrates, v. 25, n. 1, p. 60-64, 2015.

FOGAÇA, C. A.; KROHN, N. G.; SOUZA, M. A.; PAULA, R. C. Teste de tetrazólio em sementes de Copaifera langsdorffii e Schizolobium parahyba. Floresta, v. 41, n. 4, p. 895-904, 2011. 10.5380/rf.v41i4.25352.

FOGAÇA, C. A.; MALAVASI, M. M.; ZUCARELI, C.; MALAVASI, U. C. Aplicação do teste de tetrazólio em sementes de Gleditschia amorphoides Taub. Caesalpinaceae. Revista Brasileira de Sementes, v. 28, n. 3, p.101-107, 2006.

NOGUEIRA, N. W.; TORRES, S. B.; FREITAS, R. M. O. Teste de tetrazólio em sementes de timbaúba. Semina: Ciências Agrárias, v. 35, n. 6, p. 2967-2976, 2014.

PINTO, T. T. Morfoanatomia e fisiologia de sementes com dormência física de Colubrina glandulosa Perkins (Rhamnaceae) e Senna multijuga (Rich.) H. S. Irwin \& Barneby (Caesalpinioideae - Fabaceae). 2013. $71 \mathrm{f}$. Dissertação (Mestrado em Biologia de Fungos, Algas e Plantas: Área de Concentração em Fisiologia e Ecologia) Universidade Federal de Santa Catarina, Centro de Ciências Biológicas, Florianópolis, SC, 2013.

PINTO, T. L. F.; BRANCALION, P. H. S.; NOVEMBRE, A. D. L. C.; CICERO, S. M. Avaliação da viabilidade de sementes de coração-de-negro (Poecilanthe parviflora Benth. Fabaceae-faboideae) pelo teste de tetrazólio. Revista Brasileira de Sementes, v. 30, n. 1, p.208-214, 2008.

SANTOS, F. S.; VIEIRA, E. S. N.; PANOBIANCO, M. Tetrazolium test for Pinus taeda: preparation, staining, and seed viability classes. Pesquisa Agropecuária Brasileira, v. 54, e01088, 2019.

SILVA, B. A.; NOGUEIRA, J. L.; VIEIRA, E. S. N.; PANOBIANCO, M. Critérios para condução do teste de tetrazólio em sementes de araucária. Pesquisa Agropecuária Brasileira, v. 51, n. 1, p.61-68, 2016.

SILVA, F. A. S. E.; AZEVEDO, C. A. V. Versão do programa computacional Assistat para o sistema operacional Windows. Revista Brasileira de Produtos Agroindustriais, v. 4, n. 1, p. 7178, 2002.

SOUSA, D.; BRUNO, R. L. A.; SILVA, K. R. G.; TORRES, S. B.; ANDRADE, A. P. Viabilidade e vigor de sementes de Poincianella pyramidalis (Tul.) L. P. Queiroz pelo teste de tetrazólio. Revista Ciência Agronômica, v. 48, n. 2, p. 381-388, 2017. 